\title{
The relationship between serum bilirubin concentration and coronary artery ectasia
}

\author{
Mehmet Demir ${ }^{1}$, Canan Demir ${ }^{2}$, Serdar Keçeoğlu ${ }^{1}$ \\ ${ }^{1}$ Cardiology Department, Bursa Yüksek intisas Education and Research Hospital, Bursa, Turkey \\ ${ }^{2}$ Infectious Disease Department, Bursa Şevket Yılmaz Education and Research Hospital, Bursa, Turkey
}

Postep Kardiol Inter 2015; 11, 3 (41): 202-205

DOI: $10.5114 /$ pwki.2015.54014

\begin{abstract}
A bstract
Introduction: The pathophysiology of coronary artery ectasia (CAE) has not been clearly identified, although multiple abnormalities including arteritis, endothelial dysfunction, and atherothrombosis have been reported. Several studies have demonstrated that higher serum bilirubin inhibits the inflammation and proliferation of vascular smooth muscle cells; also there is a relationship between serum bilirubin and cardiovascular disease. However, the relationship between bilirubin and CAE is still unknown.

Aim: We compared serum bilirubin concentration between CAE patients and controls.

Material and methods: This study included 50 CAE patients (20 male, mean age: $60.26 \pm 10.6$ years) and 30 control persons (10 male, mean age: $57.86 \pm 11.6$ years). These participants underwent concurrent routine biochemical tests and leukocyte count, hemoglobin, hematocrit and platelet count in whole blood. These parameters were compared between groups.

Results: No statistically significant difference was found between the two groups in terms of basic characteristics. Total, direct and indirect serum bilirubin levels were significantly lower among persons with CAE patients than controls $(0.37 \pm 0.4$ vs. $0.71 \pm 0.6$ and $0.13 \pm 0.1$ vs. $0.27 \pm 0.2$ and $0.24 \pm 0.2$ vs. $0.43 \pm 0.4 \mathrm{mg} / \mathrm{dl}$; all $p<0.001$, respectively).

Conclusions: Our study revealed a relationship between serum bilirubin and CAE.
\end{abstract}

Key words: coronary artery ectasia, bilirubin, inflammation, endothelial dysfunction.

\section{Introduction}

Coronary artery ectasia (CAE) has been characterized as a localized or diffuse non-obstructive lesion of the epicardial coronary arteries with a luminal dilation exceeding 1.5-fold the normal adjacent segment or vessel diameter [1]. The prevalence of CAE varies from $1.2 \%$ to $4.7 \%$ among patients undergoing coronary angiography [2-5].

The etiopathogenesis of this coronary enlargement is completely unknown. Although the exact mechanisms leading to CAE are not clear yet, atherothrombosis, endothelial dysfunction, inflammation and vasculitis have been suggested as possible responsible factors. Coronary artery ectasia has also been reported in association with various conditions such as congenital coronary anomalies, connective tissue diseases, and vasculitis $[6,7]$.

Bilirubin is an important and potent endogen antioxidant and anti-inflammatory agent. Several previously published studies have demonstrated the relationship between serum bilirubin levels and cardiovascular dis- ease such as coronary artery disease and atrial fibrillation [8-11].

As far as we know, no study has yet been performed about the association of serum bilirubin concentration with CAE.

\section{Aim}

In our study, we compared bilirubin concentration between CAE patients and a control group.

\section{Material and methods}

The study group included 50 patients (20 male, mean age $60.26 \pm 10.6$ years) with isolated CAE who had irregularities with ectatic coronaries without any stenotic lesions under visual assessment. The control group consisted of 30 age- and gender-matched subjects (10 male, mean age $57.86 \pm 11.6$ years) who proved to have normal coronary angiograms. The indication for coronary angiography was either the presence of typical angina or pos-

\section{Corresponding author:}

Mehmet Demir, Cardiology Department, Bursa Yüksek Ihtisas Education and Research Hospital, Bursa 16100, Turkey, phone: + 90 2243605050, fax: +90 2243605055, e-mail: drmehmetdemir@hotmail.com

Received: 4.03.2014, accepted: 9.04.2014. 
itive or equivocal results of noninvasive screening tests for myocardial ischemia in both groups.

Physical examination, medical history of patients, blood biochemistry and transthoracic echocardiographic examination were evaluated in both groups to exclude systemic diseases. Patients with obstructive coronary artery disease (who had coronary stenotic lesions of $>20 \%$ ), chronic renal failure, chronic liver disorders, chronic lung disease, moderate or severe valvular disease, hypertension, diabetes mellitus, congenital heart disease, left ventricular systolic dysfunction on echocardiography (EF < 50\%), anemia, pregnancy, obstructive sleep apnea, hematological disorders, known malignancy, thyroid dysfunction, hypercholesterolemia, electrolyte imbalance, and drug history including anti-gout agents, anti-inflammatory agents (steroid or nonsteroid), antiaggregants or anticoagulant agents were excluded from the study. Also patients with a recent history of an acute infection, high body temperature $>38^{\circ} \mathrm{C}$ or an inflammatory disease were excluded from the study.

The patients having a systolic blood pressure $\geq 140$ $\mathrm{mm} \mathrm{Hg}$ and/or a diastolic blood pressure $\geq 90 \mathrm{~mm} \mathrm{Hg}$ and those taking antihypertensive drugs were accepted to be hypertensive. Diabetes was defined as a fasting blood glucose level $>126 \mathrm{mg} / \mathrm{dl}$ or current use of a diet or medication to lower blood glucose. Current cigarette smoking was defined as use of $>10$ cigarettes/day at the time of diagnosis.

\section{Coronary angiography}

Coronary angiograms were performed with a femoral approach using the Judkins technique without the use of nitroglycerin, adenosine, or a calcium channel blocker. All patients in the study population underwent elective coronary artery angiography using Siemens Axiom Artis DFC (Siemens Medical Solutions, Erlangen, Germany) following appropriate patient preparation. Coronary angiograms were judged with regard to smooth appearance, luminal wall irregularities, epicardial local or diffuse caliber reduction, and stenosis. Coronary artery ectasia was defined as dilation of the coronary artery $>1.5$-fold the diameter of the adjacent normal coronary vessels according to Falsetti and Carroll [12].

\section{Laboratory tests}

Biochemical parameters were analyzed spectrophotometrically on an Architect C16000 (Abbott, USA) autoanalyzer using an enzymatic-colorimetric assay. Also C-reactive protein (CRP) was recorded in both groups.

For whole blood count (hematocrit, hemoglobin, leukocytes, and platelets), the blood samples were collected in tubes with EDTA and analyzed on a CELL-DYN 3700 (Abbott, USA) device using the impedance and optic scatter method.

\section{Statistical analysis}

The statistical program SPSS 16.0 (SPSS Inc., Chicago, IL, USA) was used for statistical study. All values are given as mean \pm standard deviation. Mean values of continuous variables were compared between groups using the Student $t$ test or Mann-Whitney $U$ test, according to whether they were normally distributed or not, as tested by the Kolmogorov-Smirnov test. A $p$ value of less than 0.05 was considered significant.

\section{Results}

Evaluating basic clinical and demographic characteristics, there was no statistically significant difference between the two groups in terms of age, gender distribution, body mass index, and smoking status (Table I).

Serum total bilirubin, direct bilirubin and indirect bilirubin were lower in patients with CAE than controls $(0.37$ $\pm 0.4 \mathrm{mg} / \mathrm{dl}$ vs. $0.71 \pm 0.6 \mathrm{mg} / \mathrm{dl}$ and $0.13 \pm 0.1 \mathrm{mg} / \mathrm{dl}$ vs. $0.27 \pm 0.2 \mathrm{mg} / \mathrm{dl}$ and $0.24 \pm 0.2 \mathrm{mg} / \mathrm{dl}$ vs. $0.43 \pm 0.4 \mathrm{mg} / \mathrm{dl}$;

Table I. Comparison of basic clinical, biochemical and whole blood count features of patients and controls

\begin{tabular}{|c|c|c|c|}
\hline Parameter & $\begin{array}{l}\text { Patients } \\
(n=50)\end{array}$ & $\begin{array}{l}\text { Controls } \\
(n=30)\end{array}$ & Value of $p$ \\
\hline Age [years] & $60.26 \pm 10.6$ & $\begin{array}{l}57.86 \\
\pm 11.6\end{array}$ & NS \\
\hline Gender, male & $20(40)$ & $10(33)$ & NS \\
\hline Body mass index $\left[\mathrm{kg} / \mathrm{m}^{2}\right]$ & $29.8 \pm 5.4$ & $28.5 \pm 4.6$ & NS \\
\hline Smoking & $9(18)$ & $6(20)$ & NS \\
\hline Fasting glucose [mg/dl] & $95.7 \pm 9$ & $97.6 \pm 8.5$ & NS \\
\hline Creatinine $[\mathrm{mg} / \mathrm{dl}]$ & $0.75 \pm 0.1$ & $0.72 \pm 0.2$ & NS \\
\hline Total cholesterol [mg/dl] & $211 \pm 45$ & $181 \pm 36$ & NS \\
\hline Triglyceride $[\mathrm{mg} / \mathrm{dll}]$ & $162.5 \pm 65$ & $151.9 \pm 41$ & NS \\
\hline $\mathrm{TSH}[\mu \mathrm{lU} / \mathrm{ml}]$ & $1.7 \pm 0.6$ & $1.6 \pm 0.4$ & NS \\
\hline $\mathrm{CRP}[\mathrm{mg} / \mathrm{dl}]$ & $3.33 \pm 2.09$ & $2.18 \pm 1.5$ & NS \\
\hline $\mathrm{Na}[\mathrm{mmol} / \mathrm{l}]$ & $140.1 \pm 11$ & $138 \pm 9.9$ & NS \\
\hline $\mathrm{K}[\mathrm{mmol} / \mathrm{ll}]$ & $4.4 \pm 0.9$ & $4.1 \pm 0.4$ & NS \\
\hline Total bilirubin & $0.37 \pm 0.4$ & $0.71 \pm 0.6$ & $<0.001$ \\
\hline Direct bilirubin & $0.13 \pm 0.1$ & $0.27 \pm 0.2$ & $<0.001$ \\
\hline Indirect bilirubin & $0.24 \pm 0.2$ & $0.43 \pm 0.4$ & $<0.001$ \\
\hline Leukocytes $\left[10^{3} / \mu \mathrm{l}\right]$ & $9.1 \pm 6.5$ & $8.4 \pm 4.4$ & NS \\
\hline Hemoglobin $[g / d l]$ & $13.8 \pm 1.95$ & $13.49 \pm 1.15$ & NS \\
\hline Hematocrit (\%) & $40.6 \pm 3.4$ & $40.7 \pm 3.49$ & NS \\
\hline Platelet $\left[10^{3} / \mu \mathrm{I}\right]$ & $232 \pm 70$ & $234 \pm 63$ & NS \\
\hline
\end{tabular}

Results was presented as mean \pm SD or n (\%), NS - non-significant, TSH - thyroid-stimulating hormone, $C R P-C$-reactive protein. 
Table II. Logistic regression analyses

\begin{tabular}{lcc} 
Parameter & HR $(95 \% \mathrm{CI})$ & Value of $p$ \\
\hline Total bilirubin & $0.86(0.76-0.92)$ & $<0.001$ \\
\hline Direct bilirubin & $0.82(0.74-0.90)$ & $<0.001$ \\
\hline Indirect bilirubin & $0.88(0.78-0.97)$ & $<0.001$
\end{tabular}

all $p<0.001$, respectively). C-reactive protein levels were similar in both groups. Other biochemical parameters were not statistically significantly different between the 2 groups. Also there was no statistically significant difference between the two groups with regard to leukocyte count, platelet count, hemoglobin and hematocrit level (Table I).

As a result of the forward stepwise logistic regression analysis, it was found that serum total bilirubin, direct bilirubin and indirect bilirubin levels were independent predictors of CAE (Table II).

\section{Discussion}

In the present study, we found that total serum bilirubin levels were significantly lower in the CAE patient group compared to controls. To the best of our knowledge, our study is the first to focus on the relationship between bilirubin and CAE.

The pathophysiology of CAE has not been clearly identified yet, although multiple abnormalities including inflammation, endothelial dysfunction, oxidative stress, vasculitis, and atherothrombosis have been reported [5]. Coronary artery ectasia is associated with connective tissue disorders such as scleroderma, Ehlers-Danlos syndrome, and Kawasaki disease [13].

Previous studies have demonstrated that CRP, IL-6 and neutrophil/lymphocyte ratio (NLR) were higher in patients with CAE than in control participants. The increased levels of CRP and NLR may suggest that these markers may be used in clinical practice for assessment of the inflammatory status of CAE [14-16]. We found that CRP levels were higher in patients than controls, but the difference was statistically insignificant.

Recently, low serum bilirubin levels have been proposed as a useful biomarker to predict cardiovascular risk [17]. Recent evidence suggests that bilirubin acts as a potent physiologic antioxidant and anti-inflammatory agent. Recently studies have shown that elevated serum bilirubin concentrations provide important protection against atherosclerotic diseases [18, 19].

Several authors have suggested that bilirubin plays a potential role in inhibition of lipid oxidation [20,21].

Previous studies have shown that plasma bilirubin concentration is correlated inversely with several risk factors for coronary artery disease such as smoking, diabetes, and obesity, and correlated directly with HDL cholesterol [20, 22].
An inverse correlation between the presence of coronary artery disease, peripheral arterial disease, carotid intima-media thickness and bilirubin has been reported in several studies. Subnormal levels of plasma bilirubin are associated with premature coronary artery disease and cardiovascular morbidity [23, 24].

In a previous study, the 3-year incidence of coronary artery disease was significantly lower in patients with Gilbert syndrome [25].

Elevated concentrations of plasma bilirubin were suggested to be able to prevent atherogenesis. Strong ability to scavenge peroxyl radicals and the antioxidant capacity of bilirubin functioning even in a slightly increased concentration in the circulation have led to the concept that it may have a physiological function to protect against disease processes involving oxygen and peroxyl radicals [26, 27].

In a previous study, Gullu et al. found that elevated concentrations of bilirubin may serve as a protective factor in the development of coronary flow reserve impairment, coronary microvascular dysfunction, and possibly in the development of coronary atherosclerosis. They concluded that bilirubin shows the beneficial effects independent of the known coronary risk factors [28].

Induced hyperbilirubinemia was associated with a significant improvement of endothelial function in type 2 DM [29]. Also bilirubin inhibits vascular cell adhesion molecule 1 (VCAM-1) and blocks vascular smooth muscular cell proliferation [30].

As far as we know, there is no study available in the literature about the association between CAE and serum bilirubin levels. Our study is important for this reason, and we ascertained if there is an association between bilirubin and CAE.

When the 2 groups were compared in our study, serum bilirubin levels of patients having CAE were significantly lower than those of controls.

Our results may contribute to knowledge of the etiopathogenesis of CAE and pathophysiological mechanisms of increased prevalence of cardiovascular morbidity and mortality risk in these patients. The decreased concentration of bilirubin might be explained by vascular destruction, endothelial dysfunction and thrombosis in CAE patients.

\section{Conclusions}

It was found in our study that there might be an association between CAE and serum bilirubin. The measurement of bilirubin also may be used to indicate increased risk of CAE-related adverse cardiovascular events. The most important restriction of our study is the limited number of patients, and there was no obstructive coronary artery disease group. Another limitation was that angiographic diagnosis of normal coronary arteries was based on axial contrast angiograms of the vessel lumen, 
which underestimates the presence of atherosclerotic plaques. Further studies are required to determine the relation between bilirubin and CAE. We have shown for the first time that patients with CAE have lower bilirubin levels compared to controls.

\section{Conflict of interest}

The authors declare no conflict of interest.

\section{References}

1. Swaye PS, Fisher LD, Litwin P, et al. Aneurysmal coronary artery disease. Circulation 1983; 67: 134-8.

2. Hartnell GG, Parnell BM, Pridie RB. Coronary artery ectasia, its prevalence and clinical significance in 4993 patients. Br Heart J 1985; 54: 392-435.

3. Oliveros RA, Falsetti HL, Carroll RJ, et al. Arteriosclerotic coronary artery aneurysm, report of five cases and a review of the literature. Arch Intern Med 1974; 134: 1072-6.

4. Sorrell VL, Davis MJ, Bove AA. Current knowledge and significance of coronary artery ectasia: a chronologic review of the literature, recommendations for treatment, possible etiologies, and future considerations. Clin Cardiol 1998; 21: 157-60.

5. Markis JE, Joffe CD, Cohn PF, et al. Clinical significance of coronary artery ectasia. Am J Cardiol 1976; 37: 217-22.

6. Seabra-Gomes R, Somerville J, Ross DN, et al. Congenital coronary artery aneurysms. Br Heart J 1974; 36: 329-35.

7. Di Maria C, Zanchetta M, Maiolini P. Coronary artery aneurysm in a case of Ehlers-Danlos syndrome. Jpn Heart J 1998; 29: 491-6.

8. Minetti M, Mallozzi C, Di Stasi, et al. Bilirubin is an effective antioxidant of peroxynitrite-mediated protein oxidation in human blood plasma. Arch Biochem Biophys 1998; 352: 165-74.

9. Schwertner HA, Jackson WG, Tolan G. Association of low serum concentration of bilirubin with increased risk of coronary artery disease. Clin Chem 1994; 40: 18-23.

10. Levinson SS. Relationship between bilirubin, apolipoprotein B, and coronary artery disease. Ann Clin Lab Sci 1997; 27: 185-92.

11. Demir M, Demir C, Uyan U, et al. The relationship between serum bilirubin concentration and atrial fibrillation. Cardiol Res 2013; 4: 186-91.

12. Falsetti HL, Carroll RJ. Coronary artery aneurysm. A review of the literature with a report of 11 new cases. Chest 1976; 69: 630-6.

13. Mavrogeni S. Coronary artery ectasia: from diagnosis to treatment. Hellenic J Cardiol 2010; 51: 158-63.

14. Ayhan SS, Oztürk S, Erdem A, et al. Relation of neutrophil/lymphocyte ratio with the presence and severity of coronary artery ectasia. Turk Kardiyol Dern Ars 2013; 41: 185-90.

15. Turhan H, Erbay AR, Yasar AS, et al. Comparison of C-reactive protein levels in patients with coronary artery ectasia versus patients with obstructive coronary artery disease. Am J Cardiol 2004; 94: 1303-6.

16. Tokgozoglu L, Ergene O, Kinay O, et al. Plasma interleukin-6 levels are increased in coronary artery ectasia. Acta Cardiol 2004; 59: 515-9.

17. Hopkins PN, Wu LL, Hunt SC, et al. Higher serum bilirubin is associated with decreased risk for early familial coronary artery disease. Arterioscler Thromb Vasc Biol 1996; 16: 250-5.

18. Djousse L, Levy D, Cupples LA, et al. Total serum bilirubin and risk of cardiovascular disease in the Framingham offspring study. Am J Cardiol 2001; 87: 1196-200.
19. Mayer M. Association of serum bilirubin concentration with risk of coronary artery disease. Clin Chem 2000; 46: 1723-7.

20. Schwertner HA. Association of smoking and low serum bilirubin antioxidant concentrations. Atherosclerosis 1998; 136: 383-7.

21. Stocker R, Glazer AN, Ames BN. Antioxidant activity of albumin-bound bilirubin. Proc Natl Acad Sci USA 1987; 84: 5918-22.

22. Madhavan M, Wattigney WA, Srinivasan SR, et al. Serum bilirubin distribution and its relation to cardiovascular risk in children and young adults. Atherosclerosis 1997; 131: 107-13.

23. Breimer LH, Spyropolous KA, Winder AF, et al. Is bilirubin protective against coronary artery disease? Clin Chem 1994; 40 : 1987-8.

24. Ishizaka N, IshizakaY, Takahashi E, et al. High serum bilirubin level is inversely associated with the presence of carotid plaque. Stroke 2001; 32: 580-3.

25. Vitek L, Jirsa M, Brodanova M, et al. Gilbert syndrome and ischemic heart disease: a protective effect of elevated bilirubin levels. Atherosclerosis 2002; 160: 449-56.

26. Nakagami T, Toyomura K, Kinoshita T, et al. A beneficial role of bile pigments as an endogenous tissue protector: anti-complement effects of biliverdin and conjugated bilirubin. Biochim Biophys Acta 1993; 1158: 189-93.

27. Siow RC, Sato H, Mann GE. Heme oxygenase-carbon monoxide signalling pathway in atherosclerosis: anti-atherogenic actions of bilirubin and carbon monoxide? Cardiovasc Res 1999; 41: 385-494.

28. Gullu H, Erdogan D, Tok D, et al. High serum bilirubin concentrations preserve coronary flow reserve and coronary microvascular functions. Arterioscler Thromb Vasc Biol 2005; 25: 2289-94.

29. Dekker D, Dorresteijn MJ, Pijnenburg M. The bilirubin increasing drug atazanavir improves endothelial function in patients with type 2 diabetes mellitus. Arterioscler Thromb Vasc Biol 2011; 31: 458-63.

30. Ollinger R, Bilban M, Erat A, et al. Bilirubin: a natural inhibitor of vascular smooth muscle cell proliferation. Circulation 2005; 112: 1030-9. 